\title{
Asymmetric Michael Reaction of Malonate Derivatives with $\alpha, \beta$-Unsaturated Ketones Using Chiral Quaternary Ammonium Salts
}

\author{
Min Je Cho, Myoung Gi Cho, Sun Chul Huh, Sung Min Kim, Kilsung Lee, Kwang Oh Koh, \\ Joo Yang Mang, and Dae Young Kim* \\ Department of Chemistry, Soonchunhyang University, P.O. Box 97, Asan, Chungnam 336-600, Korea. *E-mail: dyoung@sch.ac.kr
${ }^{\dagger}$ Cheil Indusries Inc., Euiwang, Gyeonggi 437-010, Korea \\ Received February 24, 2006
}

\begin{abstract}
The catalytic enantioselective Michael reaction promoted by quaternary ammonium salts from Cinchona alkaloids as a phase-transfer catalyst is described. Treatment of malonates with $\alpha, \beta$-unsaturated ketones under mild reaction conditions afforded the corresponding Michael adducts in good yields with good to moderate enantiomeric excesses.
\end{abstract}

Key Words : Phase-transfer catalyst, Michael reactions, Asymmetric reactions, Malonates, Fluoromalonates

\section{Introduction}

Phase-transfer catalysis is a clean and efficient processes involving high yields, operational simplicity, mild conditions, low cost, safety, and environmental profit. ${ }^{1}$ During the past decade, the development of asymmetric phase-transfer catalysis based on the use of structurally well defined chiral, nonracemic catalysts has resulted in notable achievements, making it feasible to perform various bond formation reactions under mild phase-transfer catalyzed conditions. ${ }^{2}$

The formation of carbon-carbon bonds by conjugated addition of appropriate carboanionic reagents to $\alpha, \beta$ unsaturated carbonyl compounds is one of the most useful methods of remote functionalization in organic synthesis. ${ }^{3}$ Therefore, their catalytic asymmetric version have been studied extensively. ${ }^{4}$ Efforts toward achieving asymmetric conjugate addition of malonates to chalcones in the presence of chiral catalysts have been the subject of several reports. For example, the reaction of malonates with chalcones catalyzed by La-BINOL complexes, ${ }^{5}$ L-proline derivatives, ${ }^{6}$ chiral metal complexes, ${ }^{7}$ pyrrolidylalkyl ammonium hydroxide, ${ }^{8}$ and chiral ammonium salts. ${ }^{9}$

As part of our research program related to the development of synthetic methods for the enantioselective construction of stereogenic carbon centers, ${ }^{10}$ we reported the catalytic enantioselective Michael reaction promoted by quaternary ammonium salts from cinchonidine as a phasetransfer catalyst. ${ }^{11}$ Herein we wish to report on the catalytic asymmetric conjugate addition of malonates $\mathbf{2}$ and fluoromalonates 7 to $\alpha, \beta$-unsaturated ketones 1 using $N$-(3,5-di-tertbutyl-4-methoxy)benzylcinchonidinium bromide (4) in more details, providing information on its scopes and limitations.

\section{Results and Discussion}

Asymmetric Michael Reactions of Malonates to $\alpha, \beta$ Unsaturated Ketones. The introduction of the bulky subunit at the bridged nitrogen of Cinchona alkaloids leads to enhancement of the stereoselectivity in catalytic phase- transfer reactions. ${ }^{2}$ We introduced a bulky environment at the bridgehead nitrogen using (3,5-di-tert-butyl-4-methoxy)benzyl moiety which can be prepared in two steps starting from BHT. Phase-transfer catalysts $\mathbf{4}$ were easily prepared from cinchonine or cinchonidine. ${ }^{12}$

In order to determine suitable reaction conditions for the catalytic asymmetric conjugate addition of malonates $\mathbf{2}$ to chalcones $\mathbf{1}$, we initially investigated the reaction system using $10 \mathrm{~mol} \%$ of catalyst, with malonate as the Michael donor and chalcone 1a as the Michael acceptor (Table 1). Catalysts 4a and 4d having O-allyl group showed higher catalytic efficiencies than other catalysts in terms of yields and enantioselectivity (entries 1-5, and 13-14). It has been also found that $\mathrm{K}_{2} \mathrm{CO}_{3}$ was the more effective base in this

Table 1. Catalytic asymmetric Michael reaction of malonates 2 to chalcone 1a with phase-transfer catalysts

\begin{tabular}{|c|c|c|c|c|c|}
\hline & a & 2 & & & 3 \\
\hline entry & malonate $\mathbf{2}, \mathrm{R}$ & catalyst & base & yield $^{a}(\%)$ & $\mathrm{ee}^{b}(\%)$ \\
\hline 1 & Et & $4 a$ & $t$-BuOK & 3a, 74 & $35(R)$ \\
\hline 2 & Et & $4 b$ & $t$-BuOK & $3 a, 68$ & $13(R)$ \\
\hline 3 & $\mathrm{Et}$ & $4 c$ & $t$-BuOK & $3 a, 76$ & $22(R)$ \\
\hline 4 & Et & 4d & $t$-BuOK & $3 a, 68$ & $9(S)$ \\
\hline 5 & $\mathrm{Et}$ & $4 e$ & $t$-BuOK & 3a, 72 & $31(S)$ \\
\hline 6 & $\mathrm{Et}$ & $4 a$ & $\mathrm{KOH}$ & 3a, 74 & $40(R)$ \\
\hline $7^{c}$ & $\mathrm{Et}$ & $4 a$ & $\mathrm{KOH}$ & $3 a, 68$ & $43(R)$ \\
\hline 8 & Et & $4 a$ & $\mathrm{~K}_{2} \mathrm{CO}_{3}$ & 3a, 70 & $46(R)$ \\
\hline $9^{d}$ & Et & $4 \mathbf{a}$ & $\mathrm{K}_{2} \mathrm{CO}_{3}$ & 3a, 68 & $40(R)$ \\
\hline 10 & $\mathrm{Me}$ & $4 \mathbf{a}$ & $\mathrm{K}_{2} \mathrm{CO}_{3}$ & $\mathbf{3 b}, 92$ & $27(R)$ \\
\hline 11 & $i-\operatorname{Pr}$ & $4 a$ & $\mathrm{~K}_{2} \mathrm{CO}_{3}$ & 3c, 77 & 55 \\
\hline 12 & $\mathrm{Bn}$ & $4 a$ & $\mathrm{~K}_{2} \mathrm{CO}_{3}$ & 3d, 91 & 70 \\
\hline
\end{tabular}

${ }^{a}$ Yield of isolated product. ${ }^{b}$ Enantiopurities of $\mathbf{3 a - 3 d}$ were determined by HPLC analysis with Chiralcel AD (for 3a and 3c) and Chiralpak AS (for $\mathbf{3 b}$ and $\mathbf{3 d}$ ) columns, 2-propanol-hexane $(1: 9), 1.2 \mathrm{~mL} / \mathrm{min}, \lambda_{\max }=254$ $\mathrm{nm}$. It were established by analyses of racemic 3 that the enantiomers were fully resolved. ${ }^{c}$ Reaction carried out in $\mathrm{CH}_{2} \mathrm{Cl}_{2}$ at $0{ }^{\circ} \mathrm{C}$. ${ }^{d}$ Reaction carried out at $0{ }^{\circ} \mathrm{C}$. 
reaction than others such as $\mathrm{KOH}$ or $t$-BuOK (entries 1,6 , $8)$. Compound $(R)-\mathbf{3 a}$ was formed using cinchonine-derived catalysts (4a-4c) as the excessive enantiomer, which should be the case because all of these catalysts posses the same chirality. The cinchonidine-derived catalysts $4 \mathbf{d}-4 \mathbf{e}$ leading to formation of $(S)$-3a in excess. The absolute configuration of the major enantiomer of $\mathbf{3}$ was determined by comparison of the optical rotation and chiral HPLC analysis with those previously reported. ${ }^{5-9}$ Toluene as solvent was effective in this reaction. The reaction temperature is not critical in this reaction (entries 8 and 9). The structure of the malonate plays a key role in influencing the enantioselectivity. Dibenzyl malonate gave high selectivity in this reaction (entries 9-12).

Under the optimized reaction conditions described above (10 $\mathrm{mol} \%$ of catalyst $\mathbf{4 a}, \mathrm{K}_{2} \mathrm{CO}_{3}$, toluene, rt), we investigated catalytic asymmetric Michael reaction of dibenzyl malonate to chalcone derivatives $\mathbf{1}$. The reaction smoothly proceeded to afford the corresponding adducts $\mathbf{3}$ with good enantioselectivities. Reaction of 1.5 equiv of dibenzyl malonate with chalcone derivatives 1 , cinchonidinium salt 4 (10 $\mathrm{mol} \%)$, and $\mathrm{K}_{2} \mathrm{CO}_{3}$ in toluene at room temperature with stirring for 18-24 $\mathrm{h}$ afforded the Michael adducts 3 in good yields with moderate enantioselectivities $(45-70 \%$ ee) (Table 2$)$. In all cases the enantiomeric excesses were determined by HPLC analysis.

Having established the scope with respect to the Michael reaction of malonates with $\alpha, \beta$-unsaturated ketones, we next turned our attention to the reaction of malonates with 2cyclohexenone (Table 3 ). We subsequently tested catalysts 4a-4f. Catalyst 4d exhibited high chiral efficiency in the reaction of dibenzyl malonate with 2-cyclohexenone to afford 31 with $63 \%$ ee. Under the optimum conditions (10 mol\% of catalyst $\mathbf{4 d}, \mathrm{K}_{2} \mathrm{CO}_{3}$, toluene, $72 \mathrm{~h}$ ), the reaction of malonate derivatives with 2-cyclohexenone (11) furnished adducts 3m-3o with 67-75\% ee, respectively (Table 3, entries 7-9). Compound $(R)-31$ was formed using cinchonidinederived catalysts $(\mathbf{4 a - 4 c )}$ as the excessive enantiomer, which

Table 2. Catalytic asymmetric Michael reaction of dibenzyl malonate (2d) to chalcones $\mathbf{1}$ with phase-transfer catalyst $\mathbf{4 a}$

\begin{tabular}{|c|c|c|c|c|}
\hline 1 & $2 d$ & & & 3 \\
\hline $\mathrm{Ar}^{1}$ & $A r^{2}$ & time $(\mathrm{h})$ & yield $(\%)$ & $\mathrm{ee}^{a}(\%)$ \\
\hline $\mathrm{Ph}$ & $\mathrm{Ph}$ & 18 & 3d, 91 & 70 \\
\hline $\mathrm{Ph}$ & $p$-OMe, $\mathrm{Ph}$ & 18 & 3e, 91 & 45 \\
\hline $\mathrm{Ph}$ & 2-thienyl & 18 & 3f, 94 & 51 \\
\hline $\mathrm{Ph}$ & $p$-tolyl & 10 & 3g, 88 & 47 \\
\hline $\mathrm{Ph}$ & 2-furyl & 10 & 3h, 92 & 49 \\
\hline$p$-OMe, $\mathrm{Ph}$ & $\mathrm{Ph}$ & 18 & $3 \mathbf{i}, 88$ & 47 \\
\hline 2-naphthyl & $m-\mathrm{Br}, \mathrm{Ph}$ & 24 & $3 \mathbf{j}, 60$ & 67 \\
\hline 2-naphthyl & $p$-Br, $\mathrm{Ph}$ & 24 & $\mathbf{3 k}, 58$ & 59 \\
\hline
\end{tabular}

${ }^{a}$ Enantiopurities of $\mathbf{3 d - 3 k}$ were determined by HPLC analysis with chiral column (Chiralpak AS for $\mathbf{3 d - 3 i}$, Chiralcel OD-H for $\mathbf{3 j}$ and $\mathbf{3 k}$ ), 2 propanol-hexane $(1: 9), 1.2 \mathrm{~mL} / \mathrm{min}, \lambda_{\max }=254 \mathrm{~nm}$. In each case, it was established by analyses of racemic 3 that the enantiomers were fully resolved.
Table 3. Catalytic asymmetric Michael reaction of malonate 2 to 2cyclohexenone (11) with phase-transfer catalysts 4

\begin{tabular}{|c|c|c|c|c|}
\hline 11 & $\mathrm{R}^{2} \gamma_{2}^{\mathrm{COOR}}$ & $\begin{array}{c}\text { catal } \\
\mathrm{K}_{2} \mathrm{CO}_{3} \\
\mathrm{rt},\end{array}$ & & \\
\hline entry & $\begin{array}{c}\text { malonate } 2 \\
\mathrm{R}^{1}, \mathrm{R}^{2}\end{array}$ & catalyst & yield (\%) & $\mathrm{ee}^{a, b}(\%)$ \\
\hline 1 & $\mathrm{Bn}, \mathrm{H}$ & $4 a$ & 31,66 & $33(R)$ \\
\hline 2 & $\mathrm{Bn}, \mathrm{H}$ & $4 b$ & 31,76 & $17(R)$ \\
\hline 3 & $\mathrm{Bn}, \mathrm{H}$ & $4 c$ & 31,60 & $29(R)$ \\
\hline 4 & $\mathrm{Bn}, \mathrm{H}$ & 4d & 31, 74 & $63(S)$ \\
\hline 5 & $\mathrm{Bn}, \mathrm{H}$ & $4 e$ & 31,65 & $27(S)$ \\
\hline 6 & $\mathrm{Bn}, \mathrm{H}$ & $4 f$ & 31, 74 & $43(S)$ \\
\hline 7 & $\mathrm{Me}, \mathrm{H}$ & 4d & $3 \mathbf{m}, 73$ & $67(S)$ \\
\hline 8 & Et, H & $4 d$ & $3 \mathbf{n}, 83$ & $75(S)$ \\
\hline 9 & $\mathrm{Bn}, \mathrm{Me}$ & $4 d$ & 3o, 76 & $73(S)$ \\
\hline
\end{tabular}

${ }^{a}$ Enantiopurities of 31-3o were determined by HPLC analysis with Chiralpak AS, 2-propanol-hexane (1:9), $1.2 \mathrm{~mL} / \mathrm{min}, \lambda_{\max }=215 \mathrm{~nm}$. ${ }^{b}$ The absolute configuration was established through comparison of its optical rotation and HPLC data with chiral columns to that reported in the literature. ${ }^{5,7}$

should be the case because all of these catalysts posses the same chirality. The cinchonine-derived catalysts $\mathbf{4 d - 4 f}$ leading to formation of $(S)-31$ in excess.

Asymmetric Michael reactions of fluoromalonates to chalcones. Organic fluorine compounds are of importance in organic synthesis because of their use as medicinals, agrochemicals, and in fundamental studies of biochemical and metabolic process. ${ }^{13}$ However, enantioselective Michael reactions of fluoromalonate to $\alpha, \beta$-unsaturated carbonyl compounds have scarcely been studied. In analogy with above reaction previously studied, we initially investigated the reaction system using $10 \mathrm{~mol} \%$ of catalyst, with diethyl fluoromalonate 7a as the Michael donor and chalcone 1a as the Michael acceptor.

To examine the generality of the enantioselective Michael reaction using chiral phase-transfer catalysts, we investigated catalytic asymmetric Michael reaction of diethyl fluoromalonate 7a to chalcone derivatives 1a under the optimized reaction conditions described above $(10 \mathrm{~mol} \%$ of catalyst 4a, $\mathrm{K}_{2} \mathrm{CO}_{3}$, toluene, rt). The reaction smoothly proceeded to afford the corresponding adduct $\mathbf{8}$ with moderate enantioselectivities (Table 4). Reaction of 1.0 equiv of diethyl fluoromalonate 2 with chalcone derivatives

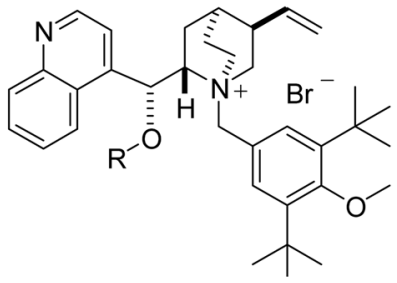

4a, $R=$ allyl

4b, $R=$ benzyl

4c, $R=$ propagyl

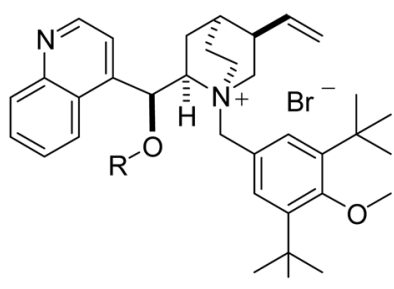

4d, $R=$ allyl

4e, $R=$ benzyl

4f, $R$ = propagy
Figure 1. Chiral phase-transfer catalysts. 
Table 4. Catalytic asymmetric Michael reaction of diethyl fluoromalonates $\mathbf{7}$ to chalcones $\mathbf{1}$ with phase-transfer catalyst $\mathbf{4 a}$

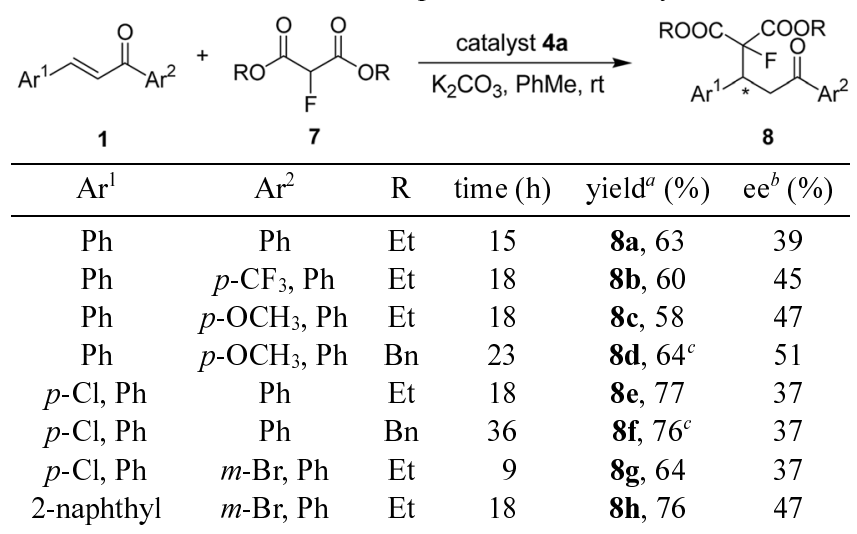

${ }^{a}$ Isolated yields are based on chalcone. ${ }^{b}$ Enantiomeric excess was determined by HPLC analysis using Chiralcel OD-H (for $\mathbf{8 a}, \mathbf{8 b}, \mathbf{8 e}, \mathbf{8 g}$, and $\mathbf{8 h}$ ), AS (for 8d and 8f), and Whelk-O1 (for 8c) columns. ${ }^{c}$ Reaction carried out using $\mathrm{Rb}_{2} \mathrm{CO}_{3}$ as base.

1, cinchonidinium salt $4 \mathbf{a}(10 \mathrm{~mol} \%)$, and $\mathrm{K}_{2} \mathrm{CO}_{3}$ in toluene at room temperature with stirring for $15-18 \mathrm{~h}$ afforded the Michael adducts $\mathbf{8}$ in good yields with moderate enantioselectivities $(35-47 \%$ ee) (Table 2$)$.

In conclusion, we have developed a new class of asymmetric phase-transfer catalyst, which shows good enantioselectivity in the Michael reaction of malonate and fluoromalonate to $\alpha, \beta$-unsaturated ketones. We are currently involved in the further development of these catalyst systems and investigating their applicability to other asymmetric phase-transfer processes.

\section{Experimental Section}

General. All reactions were carried out in oven-dried glassware under an atmosphere of dry nitrogen unless otherwise noted. All reaction were magnetically stirred and monitored by analytical thin layer chromatography using Merck pre-coated silica gel plates with $\mathrm{F}_{254}$ indicator. Flash column chromatography was performed using silicagel 60 (mesh 230-400) supplied by E. Merck. ${ }^{1} \mathrm{H}$ NMR and ${ }^{13} \mathrm{C}$ NMR spectra were recorded on a Bruker AC $200(200 \mathrm{MHz}$ for ${ }^{1} \mathrm{H}, 50 \mathrm{MHz}$ for ${ }^{13} \mathrm{C}$ ) or Bruker DRS 400 (400 MHz for ${ }^{1} \mathrm{H}, 100 \mathrm{MHz}$ for $\left.{ }^{13} \mathrm{C}\right)$. Chemical shift values $(\delta)$ are reported in ppm relative to $\mathrm{Me}_{4} \mathrm{Si}(\delta 0.0 \mathrm{ppm})$. Multiplicities are indicated by s (singlet), d (doublet), $\mathrm{t}$ (triplet), q (quartet), $\mathrm{m}$ (multiplet) and br (broad). Mass spectra were measured on a Jeol HX110/110A using electrospray ionization technique. Optical rotations were measured with a JASCO-DIP-1000 digital polarimeter. High-performance liquid chromatography (HPLC) was performed on a Younglin M930 Series equipped with variable wavelength detector using chiral stationary column (250 mm, $4.6 \mathrm{~mm})$ such as Chiralpak AS, Chiralcel OD-H, and Whelk O1 columns.

General Procedure for Michael Addition of Malonates to Chalcones. A mixture of malonate derivatives $(0.45$ $\mathrm{mmol}), \mathrm{K}_{2} \mathrm{CO}_{3}(0.16 \mathrm{~g}, 2.0 \mathrm{mmol})$, chiral cinchonidinium salt $4 \mathbf{a}(18.0 \mathrm{mg}, 0.03 \mathrm{mmol})$, and chalcones $(0.3 \mathrm{mmol})$ in toluene $(2 \mathrm{~mL})$ was stirred at room temperature for 9-24 h. The mixture was diluted with water $(10 \mathrm{~mL})$ and extracted with ethyl acetate $(2 \times 10 \mathrm{~mL})$. The combined organic layers were dried over $\mathrm{MgSO}_{4}$, filtered, concentrated, and purified by flash chromatography (silica gel, ethyl acetate : hexane = $1: 5)$ to afford Michael adducts.

2-(3-Oxo-1,3-diphenylpropyl)dibenzylmalonate (3d). ${ }^{9 b}$ $\mathrm{R}_{\mathrm{f}} 0.32($ EtOAc : hexane $=1: 5) ;[\alpha]_{\mathrm{D}}^{20}-25.9\left(\mathrm{c} 1.0 \mathrm{CHCl}_{3}\right.$, $70 \%$ ee); ${ }^{1} \mathrm{H} \mathrm{NMR}\left(\mathrm{CDCl}_{3}, 200 \mathrm{MHz}\right) \delta 3.42-3.45(\mathrm{~m}, 2 \mathrm{H})$, $3.94(\mathrm{~d}, J=9.6 \mathrm{~Hz}, 1 \mathrm{H}), 4.16-4.28(\mathrm{~m}, 1 \mathrm{H}), 4.91(\mathrm{~s}, 2 \mathrm{H})$, $5.14(\mathrm{~s}, 2 \mathrm{H}), 7.03-7.83(\mathrm{~m}, 20 \mathrm{H}) ;{ }^{13} \mathrm{C} \mathrm{NMR}\left(\mathrm{CDCl}_{3}, 50\right.$ MHz) $\delta 197.3,167.9,167.4,140.2,136.6,135.0,133.0$, 128.4, 128.3, 128.1, 128.0, 127.1, 67.3, 67.1, 57.4, 42.2, 40.7; $\mathrm{R}_{\mathrm{t}}$ HPLC (90 : 10, hexane : iso-PrOH, $254 \mathrm{~nm}, 0.5 \mathrm{~mL} /$ min) Chiralpak AS column, $t_{R}=45.2 \min ($ minor $), t_{R}=49.5$ $\min$ (major).

2-[3-(4-Methoxyphenyl)-3-oxo-1-phenylpropyl]dibenzylmalonate (3e). $\mathrm{R}_{\mathrm{f}} 0.26($ EtOAc : hexane $=1: 5) ;[\alpha]_{\mathrm{D}}^{25}-5.9$ (c $1.0 \mathrm{CHCl}_{3}, 45 \%$ ee); ${ }^{1} \mathrm{H}$ NMR $\left(\mathrm{CDCl}_{3}, 200 \mathrm{MHz}\right) \delta 3.37-$ 3.48 (m, 2H), 3.73 (s, 3H), 3.89 (d, J=17.7 Hz, 1H), 4.13$4.22(\mathrm{~m}, 1 \mathrm{H}), 4.91(\mathrm{~s}, 2 \mathrm{H}), 5.15(\mathrm{~s}, 2 \mathrm{H}), 6.69-7.82(\mathrm{~m}, 19 \mathrm{H})$; ${ }^{13} \mathrm{C} \mathrm{NMR}\left(\mathrm{CDCl}_{3}, 50 \mathrm{MHz}\right) \delta 197.5,168.0,167.5,136.6$, $135.1,132.9,132.0,129.1,128.4,128.3,128.1,128.0$, 113.7, 67.2, 67.0, 57.7, 55.0, 42.4, 40.1; $\mathrm{R}_{\mathrm{t}} \operatorname{HPLC}(90: 10$, hexane : iso-PrOH, $254 \mathrm{~nm}, 0.5 \mathrm{~mL} / \mathrm{min})$ Chiralpak AS column, $\mathrm{t}_{\mathrm{R}}=36.4 \mathrm{~min}$ (minor), $\mathrm{t}_{\mathrm{R}}=39.5 \mathrm{~min}$ (major).

2-(3-Oxo-1-phenyl-3-thiophen-2-yl-propyl)dibenzylmalonate (3f). $\mathrm{R}_{\mathrm{f}} 0.35$ (EtOAc : hexane $\left.=1: 5\right) ;[\alpha]_{\mathrm{D}}^{25}-8.3$ (c $1.1 \mathrm{CHCl}_{3}, 51 \%$ ee); ${ }^{1} \mathrm{H}$ NMR $\left(\mathrm{CDCl}_{3}, 200 \mathrm{MHz}\right) \delta 3.34-$ $3.38(\mathrm{~m}, 2 \mathrm{H}), 3.96(\mathrm{~d}, J=11.2 \mathrm{~Hz}, 1 \mathrm{H}), 4.17-4.22(\mathrm{~m}, 1 \mathrm{H})$, 4.89 (s, 2H), 5.15 (d, $J=2.2 \mathrm{~Hz}, 2 \mathrm{H}), 7.04-7.63(\mathrm{~m}, 18 \mathrm{H})$; ${ }^{13} \mathrm{C} \mathrm{NMR}\left(\mathrm{CDCl}_{3}, 50 \mathrm{MHz}\right) \delta 195.3,167.9,167.3,139.8$, 134.9, 133.6, 132.0, 128.5, 128.4, 128.2, 128.1, 127.2, 67.3, 67.1, 57.3, 42.9, 41.0; $\mathrm{R}_{\mathrm{t}}$ HPLC (90 : 10, hexane : iso-PrOH, $254 \mathrm{~nm}, 1.2 \mathrm{~mL} / \mathrm{min}$ ) Chiralpak AS column, $\mathrm{t}_{\mathrm{R}}=15.8 \mathrm{~min}$ (minor), $\mathrm{t}_{\mathrm{R}}=16.8 \mathrm{~min}$ (major).

2-(3-Oxo-1-phenyl-3-p-tolylpropyl)dibenzylmalonate (3g). $\mathrm{R}_{\mathrm{f}} 0.32($ EtOAc : hexane $=1: 5) ;[\alpha]_{\mathrm{D}}^{20}-11.4\left(\mathrm{c} 1.0 \mathrm{CHCl}_{3}\right.$, $49 \%$ ee); ${ }^{1} \mathrm{H}$ NMR $\left(\mathrm{CDCl}_{3}, 200 \mathrm{MHz}\right) \delta 2.38(\mathrm{~s}, 3 \mathrm{H}), 3.44-$ $3.55(\mathrm{~m}, 1 \mathrm{H}), 3.99$ (d, $J=10.2 \mathrm{~Hz}, 1 \mathrm{H}), 4.16-4.21(\mathrm{~m}, 1 \mathrm{H})$, 4.92 (s, 2H), 5.16 (d, $J=7.4 \mathrm{~Hz}, 2 \mathrm{H}), 7.03-7.87$ (m, 19H); ${ }^{13} \mathrm{C} \mathrm{NMR}\left(\mathrm{CDCl}_{3}, 50 \mathrm{MHz}\right) \delta 196.9,167.9,167.4,128.5$, 128.4, 128.2, 128.1, 127.1, 67.2, 67.0, 57.5, 42.1, 40.7; $\mathrm{R}_{\mathrm{t}}$ HPLC (90 : 10, hexane : iso-PrOH, $254 \mathrm{~nm}, 1.2 \mathrm{~mL} / \mathrm{min}$ ) Chiralpak AS column, $t_{R}=10.5$ min (major), $t_{R}=12.7 \mathrm{~min}$ (minor).

2-(3-Furan-2-yl-3-oxo-1-phenylpropyl)dibenzylmalonate (3h). $\mathrm{R}_{\mathrm{f}} 0.35$ (EtOAc : hexane $\left.=1: 5\right) ;{ }^{1} \mathrm{H} \mathrm{NMR}\left(\mathrm{CDCl}_{3}\right.$, $200 \mathrm{MHz}) \delta 3.26-3.36(\mathrm{~m}, 1 \mathrm{H}), 3.94(\mathrm{~d}, J=11.2 \mathrm{~Hz}, 1 \mathrm{H})$, 4.13-4.22 (m, 1H), 4.89 (s, 2H), 5.14 (d, $J=1.8 \mathrm{~Hz}, 2 \mathrm{H})$, 6.44-6.47 (m, 1H), 7.02-7.59 (m, 17H); ${ }^{13} \mathrm{C} \mathrm{NMR}\left(\mathrm{CDCl}_{3}\right.$, $50 \mathrm{MHz}) \delta 186.3,167.7,167.3,146.2,139.9,134.9,128.4$, 128.1, 127.2, 117.1, 112.1, 67.3, 67.1, 57.3, 42.1, 40.6; $\mathrm{R}_{\mathrm{t}}$ HPLC (90 : 10, hexane : iso-PrOH, $254 \mathrm{~nm}, 1.2 \mathrm{~mL} / \mathrm{min}$ ) Chiralpak AS column, $t_{R}=18.2 \mathrm{~min}($ minor $), t_{R}=20.2 \mathrm{~min}$ (major).

2-[1-(4-Methoxyphenyl)-3-oxo-3-phenylpropyl]dibenzyl- 
malonate (3i). $\mathrm{R}_{\mathrm{f}} 0.32($ EtOAc : hexane $=1: 5) ;[\alpha]_{\mathrm{D}}^{25}$ -10.8 (c $2.1 \mathrm{CHCl}_{3}, 47 \%$ ee); ${ }^{1} \mathrm{H} \mathrm{NMR}\left(\mathrm{CDCl}_{3}, 200 \mathrm{MHz}\right)$ $\delta 3.36-3.45(\mathrm{~m}, 2 \mathrm{H}), 3.85(\mathrm{~s}, 3 \mathrm{H}), 3.94(\mathrm{~d}, J=9.5 \mathrm{~Hz}, 1 \mathrm{H})$, 4.15-4.23 (m, 1H), 4.90 (s, 2H), 5.13 (d, $J=2.2 \mathrm{~Hz}, 2 \mathrm{H})$, 6.83-7.82 (m, 19H); ${ }^{13} \mathrm{C} \mathrm{NMR}\left(\mathrm{CDCl}_{3}, 50 \mathrm{MHz}\right) \delta 196.7$, 168.7, 167.7, 130.2, 128.4, 127.1, 113.5, 67.2, 67.0, 57.5, 55.4, 41.9, 40.9; $\mathrm{R}_{\mathrm{t}}$ HPLC (90 : 10, hexane : iso-PrOH, 254 $\mathrm{nm}, 1.2 \mathrm{~mL} / \mathrm{min})$ Chiralpak $\mathrm{AS}$ column, $\mathrm{t}_{\mathrm{R}}=18.5 \mathrm{~min}$ (minor), $\mathrm{t}_{\mathrm{R}}=24.9 \mathrm{~min}$ (major).

2-[3-(3-Bromophenyl)-1-naphthalen-2-yl-3-oxopropyl]dibenzylmalonate $(\mathbf{3 j}) . \mathrm{R}_{\mathrm{f}} 0.25$ (EtOAc : hexane $=1$ : 5); $[\alpha]_{\mathrm{D}}^{25}-9.0\left(\mathrm{c} 1.0 \mathrm{CHCl}_{3}, 67 \%\right.$ ee); ${ }^{1} \mathrm{H} \mathrm{NMR}\left(\mathrm{CDCl}_{3}, 200\right.$ MHz) $\delta 3.40-3.47(\mathrm{~m}, 2 \mathrm{H}), 4.04(\mathrm{~d}, J=9.79 \mathrm{~Hz}, 1 \mathrm{H}), 4.33-$ $4.37(\mathrm{~m}, 1 \mathrm{H}), 4.87$ (s, 2H), $5.22(\mathrm{~d}, J=2.0 \mathrm{~Hz}, 2 \mathrm{H}), 6.88-$ $7.73(\mathrm{~m}, 21 \mathrm{H}) ;{ }^{13} \mathrm{C} \mathrm{NMR}\left(\mathrm{CDCl}_{3}, 50 \mathrm{MHz}\right) \delta 197.7,167.9$, $167.4,137.5,131.7,129.5,128.5,128.4,128.2,128.0$, $127.8,127.5,127.0,126.0,125.8,67.4,67.2,57.4,42.2$, 40.8; $\mathrm{R}_{\mathrm{t}}$ HPLC (90 : 10, hexane : iso-PrOH, $254 \mathrm{~nm}, 1.2 \mathrm{~mL} /$ min) Chiralcel OD-H column, $t_{R}=29.8 \min ($ major $), t_{R}=$ $46.9 \mathrm{~min}$ (minor).

2-[3-(4-Bromophenyl)-1-naphthalen-2-yl-3-oxopropyl] dibenzylmalonate (3k). $\mathrm{R}_{\mathrm{f}} 0.25$ (EtOAc : hexane $=1: 5$ ); $[\alpha]_{\mathrm{D}}^{22}-6.1$ (c $1.0 \mathrm{CHCl}_{3}, 59 \%$ ee); ${ }^{1} \mathrm{H} \mathrm{NMR}\left(\mathrm{CDCl}_{3}, 200\right.$ MHz) $\delta 3.46-3.49(\mathrm{~m}, 2 \mathrm{H}), 4.02(\mathrm{~d}, J=9.6 \mathrm{~Hz}, 1 \mathrm{H}), 4.33-$ $4.41(\mathrm{~m}, 1 \mathrm{H}), 4.95(\mathrm{~s}, 2 \mathrm{H}), 5.15(\mathrm{~d}, J=2.0 \mathrm{~Hz}, 2 \mathrm{H}), 6.88-$ $7.74(\mathrm{~m}, 21 \mathrm{H}) ;{ }^{13} \mathrm{C} \mathrm{NMR}\left(\mathrm{CDCl}_{3}, 50 \mathrm{MHz}\right) \delta 194.4,167.9$, $167.1,131.7,129.5,128.5,128.4,128.2,128.0,127.8$, 127.5, 127.0, 126.0, 125.8, 67.4, 67.2, 57.4, 42.2, 40.8; $\mathrm{R}_{\mathrm{t}}$ HPLC (90 : 10, hexane : iso-PrOH, $254 \mathrm{~nm}, 1.2 \mathrm{~mL} / \mathrm{min}$ ) Chiralcel OD-H column, $t_{R}=26.9 \min$ (major), $t_{R}=36.3$ $\min$ (minor).

(S)-3-[Bis(benzyloxycarbonyl)methyl]cyclohexanone (3I). ${ }^{5 \mathrm{e}, 7 \mathrm{e}} \mathrm{R}_{\mathrm{f}} 0.3$ (EtOAc : hexane $=1: 4$ ); $[\alpha]_{\mathrm{D}}^{22}-1.4$ (c 1.5 $\mathrm{CHCl}_{3}, 63 \%$ ee); ${ }^{1} \mathrm{H}$ NMR $\left(\mathrm{CDCl}_{3}, 200 \mathrm{MHz}\right) \delta 1.42-1.63$ $(\mathrm{m}, 2 \mathrm{H}), 1.86-2.62(\mathrm{~m}, 7 \mathrm{H}), 3.41(\mathrm{~d}, J=7.8 \mathrm{~Hz}, 1 \mathrm{H}), 5.14(\mathrm{~s}$, $2 \mathrm{H}), 5.15(\mathrm{~s}, 2 \mathrm{H}), 7.28-7.40(\mathrm{~m}, 10 \mathrm{H}) ;{ }^{13} \mathrm{C} \mathrm{NMR}\left(\mathrm{CDCl}_{3}, 50\right.$ MHz) $\delta 24.5,28.6,38.1,40.9,45.0,56.7,67.3,128.3,128.5$, 128.6, 135.1, 167.5, 209.4; $\mathrm{R}_{\mathrm{t}}$ HPLC (90 : 10, hexane : iso$\mathrm{PrOH}, 215 \mathrm{~nm}, 1.2 \mathrm{~mL} / \mathrm{min}$ ) Chiralcel AS column, $\mathrm{t}_{\mathrm{R}}=23.9$ (minor), $\mathrm{t}_{\mathrm{R}}=29.1$ (major).

(S)-3-[Bis(methyloxycarbonyl)methyl]cyclohexanone (3m). ${ }^{5 \mathrm{~d}, 5 \mathrm{e}} \mathrm{R}_{\mathrm{f}} 0.3($ EtOAc : hexane $=1: 2) ;[\alpha]_{\mathrm{D}}^{22}-2.7$ (c 2.5 $\mathrm{CHCl}_{3}, 67 \%$ ee); ${ }^{1} \mathrm{H} \mathrm{NMR}\left(\mathrm{CDCl}_{3}, 200 \mathrm{MHz}\right) \delta 1.46-1.82$ $(\mathrm{m}, 2 \mathrm{H}), 1.92-2.51(\mathrm{~m}, 7 \mathrm{H}), 3.36(\mathrm{~d}, J=7.8 \mathrm{~Hz}, 1 \mathrm{H}), 3.76(\mathrm{~d}$, $J=1.7 \mathrm{~Hz}, 6 \mathrm{H}) ;{ }^{13} \mathrm{C} \mathrm{NMR}\left(\mathrm{CDCl}_{3}, 50 \mathrm{MHz}\right) \delta 24.5,28.7$, 38.0, 40.9, 45.0, 52.5, 56.6, 168.2, 209.4; $\mathrm{R}_{\mathrm{t}} \operatorname{HPLC}(90$ : 10, hexane : iso-PrOH, $215 \mathrm{~nm}, 1.2 \mathrm{~mL} / \mathrm{min})$ Chiralcel AS column, $\mathrm{t}_{\mathrm{R}}=20.5$ (minor), $\mathrm{t}_{\mathrm{R}}=26.3$ (major).

(S)-3-[Bis(ethyloxycarbonyl)methyl]cyclohexanone (3n). ${ }^{\text {7d }}$ $\mathrm{R}_{\mathrm{f}} 0.3$ (EtOAc : hexane $\left.=1: 5\right) ;[\alpha]_{\mathrm{D}}^{20}-2.2\left(\mathrm{c} 1.7 \mathrm{CHCl}_{3}\right.$, $75 \%$ ee); ${ }^{1} \mathrm{H} \mathrm{NMR}\left(\mathrm{CDCl}_{3}, 200 \mathrm{MHz}\right) \delta 1.28(\mathrm{t}, J=7.3 \mathrm{~Hz}$, $6 \mathrm{H}), 1.19-1.66(\mathrm{~m}, 2 \mathrm{H}), 2.01-2.50(\mathrm{~m}, 7 \mathrm{H}), 3.30$ (d, $J=7.9$ $\mathrm{Hz}, 1 \mathrm{H}), 4.15-4.27(\mathrm{~m}, 4 \mathrm{H}) ;{ }^{13} \mathrm{C} \mathrm{NMR}\left(\mathrm{CDCl}_{3}, 50 \mathrm{MHz}\right)$ $\delta 14.1,24.5,28.8,38.0,41.0,45.1,56.9,60.4,61.5,167.86$, 209.7; R $\mathrm{R}_{\mathrm{t}}$ HPLC (90 : 10, hexane : iso-PrOH, $215 \mathrm{~nm}, 1.2$ $\mathrm{mL} / \mathrm{min}$ ) Chiralcel AS column, $t_{\mathrm{R}}=12.3$ (minor), $\mathrm{t}_{\mathrm{R}}=14.7$ (major).
(S)-3-[Bis(benzyloxycarbonyl)ethyl]cyclohexanone (3o). ${ }^{5 \mathrm{~d}}$ $\mathrm{R}_{\mathrm{f}} 0.25$ (EtOAc : hexane $\left.=1: 4\right) ;[\alpha]_{\mathrm{D}}^{20}-0.4\left(\mathrm{c} 1.8 \mathrm{CHCl}_{3}\right.$, $73 \%$ ee); ${ }^{1} \mathrm{H}$ NMR $\left(\mathrm{CDCl}_{3}, 200 \mathrm{MHz}\right) \delta 1.35-1.83(\mathrm{~m}, 2 \mathrm{H})$, $1.44(\mathrm{~s}, 3 \mathrm{H}), 1.97-2.63(\mathrm{~m}, 7 \mathrm{H}), 5.11(\mathrm{~s}, 4 \mathrm{H}), 7.21-7.38(\mathrm{~m}$, $10 \mathrm{H}) ;{ }^{13} \mathrm{C} \mathrm{NMR}\left(\mathrm{CDCl}_{3}, 50 \mathrm{MHz}\right) \delta 16.8,24.6,26.6,41.0$, 42.6, 43.2, 57.0, 67.1, 67.2, 128.1, 128.6, 128.5, 135.2, 170.5, 210.0; $\mathrm{R}_{\mathrm{t}}$ HPLC (90: 10, hexane : iso-PrOH, $215 \mathrm{~nm}$, $1.2 \mathrm{~mL} / \mathrm{min}$ ) Chiralcel AS column, $\mathrm{t}_{\mathrm{R}}=11.2$ (minor), $\mathrm{t}_{\mathrm{R}}=$ 19.0 (major).

2-Fluoro-2-(3-oxo-1,3-diphenylpropyl)diethylmalonate (8a). $\mathrm{R}_{\mathrm{f}} 0.25($ EtOAc : hexane $=1: 6) ;[\alpha]_{\mathrm{D}}^{25}-16.3(\mathrm{c}=1.0$, $\mathrm{CHCl}_{3}, 39 \%$ ee); ${ }^{1} \mathrm{H} \mathrm{NMR}\left(\mathrm{CDCl}_{3}, 200 \mathrm{MHz}\right) \delta 1.03(\mathrm{t}, J=$ $7.2 \mathrm{~Hz}, 3 \mathrm{H}), 1.31$ (t, $J=7.3 \mathrm{~Hz}, 3 \mathrm{H}), 3.38(\mathrm{dd}, J=3.6,17.6$ $\mathrm{Hz}, 1 \mathrm{H}), 3.68$ (dd, $J=9.6,17.5 \mathrm{~Hz}, 1 \mathrm{H}), 3.95-4.09$ (m, 2H), $4.32(\mathrm{q}, J=7.2 \mathrm{~Hz}, 2 \mathrm{H}), 4.54(\mathrm{ddd}, J=3.6,9.6,32.9 \mathrm{~Hz}$, 1H), 7.24 (d, $J=7.8 \mathrm{~Hz}, 2 \mathrm{H}), 7.36-7.46(\mathrm{~m}, 5 \mathrm{H}), 7.55$ (d, $J=$ $9.5 \mathrm{~Hz}, 1 \mathrm{H}), 7.90(\mathrm{~d}, J=5.3 \mathrm{~Hz}, 2 \mathrm{H}) ;{ }^{13} \mathrm{C} \mathrm{NMR}\left(\mathrm{CDCl}_{3}, 50\right.$ MHz) $\delta 197.3,167.9,167.4,140.2,136.6,135.0,133.0$, 128.4, 128.3, 128.1, 128.0, 127.1, 67.3, 67.1, 57.4, 42.2, 40.7; HRMS calcd for $\mathrm{C}_{22} \mathrm{H}_{23} \mathrm{FO}_{5} \mathrm{Na}\left([\mathrm{M}+\mathrm{Na}]^{+}\right) 409.1427$, found 409.1425; $\mathrm{R}_{\mathrm{t}}$ HPLC (90:10, hexane : iso-PrOH, 254 $\mathrm{nm}, 0.5 \mathrm{~mL} / \mathrm{min}$ ) Chiralcel OD-H column, $\mathrm{t}_{\mathrm{R}}=14.0 \mathrm{~min}$ (major), $\mathrm{t}_{\mathrm{R}}=15.5 \mathrm{~min}$ (minor).

2-Fluoro-2-[3-oxo-1-phenyl-3-(4-trifluoromethylphenyl) propyl]diethylmalonate $(\mathbf{8 b}) . \mathrm{R}_{\mathrm{f}} 0.29(\mathrm{EtOAc}$ : hexane $=$ $1: 6) ;[\alpha]_{\mathrm{D}}^{25}-13.5\left(\mathrm{c}=1.0, \mathrm{CHCl}_{3}, 45 \%\right.$ ee $) ;{ }^{1} \mathrm{H} \mathrm{NMR}$ $\left(\mathrm{CDCl}_{3}, 400 \mathrm{MHz}\right) \delta 1.02(\mathrm{t}, J=7.2 \mathrm{~Hz}, 3 \mathrm{H}), 1.32(\mathrm{t}, J=7.2$ $\mathrm{Hz}, 3 \mathrm{H}), 3.43$ (dd, $J=4.0,17.2 \mathrm{~Hz}, 1 \mathrm{H}), 3.64$ (dd, $J=9.6$, $17.2 \mathrm{~Hz}, 1 \mathrm{H}), 3.93-4.06(\mathrm{~m}, 2 \mathrm{H}), 4.31$ (q, $J=7.2 \mathrm{~Hz}, 2 \mathrm{H})$, 4.49 (ddd, $J=3.6,9.6,32.4 \mathrm{~Hz}, 1 \mathrm{H}), 7.19-7.25$ (m, 3H), $7.34(\mathrm{~d}, J=8.4 \mathrm{~Hz}, 2 \mathrm{H}), 7.67(\mathrm{~d}, J=8.0 \mathrm{~Hz}, 2 \mathrm{H}), 7.97(\mathrm{~d}$, $J=8.0 \mathrm{~Hz}, 2 \mathrm{H}) ;{ }^{13} \mathrm{C} \mathrm{NMR}\left(\mathrm{CDCl}_{3}, 50 \mathrm{MHz}\right) \delta 196.3,167.9$, 167.3, 139.0, 137.4, 135.2, 134.9, 134.7, 133.1, 132.5, 131.7, $128.9,128.5,128.3,128.2,128.1,128.0,127.8,127.5,126.9$, 126.0, 125.7, 67.3, 67.1, 57.3, 42.2, 40.8; HRMS calcd for $\mathrm{C}_{23} \mathrm{H}_{22} \mathrm{~F}_{4} \mathrm{O}_{5} \mathrm{Na}\left([\mathrm{M}+\mathrm{Na}]^{+}\right)$477.1301, found 477.1307; $\mathrm{R}_{\mathrm{t}}$ HPLC (90 : 10, hexane : iso-PrOH, $254 \mathrm{~nm}, 0.5 \mathrm{~mL} / \mathrm{min}$ ) Chiralcel OD-H column, $t_{R}=13.7$ min (major), $t_{R}=19.7$ $\min$ (minor).

2-Fluoro-2-[3-(4-methoxyphenyl)-3-oxo-1-phenylpropyl] diethylmalonate $(\mathbf{8 c}) . \mathrm{R}_{\mathrm{f}} 0.13($ EtOAc : hexane $=1$ : 6); $[\alpha]_{\mathrm{D}}^{25}-7.4\left(\mathrm{c}=1.0, \mathrm{CHCl}_{3}, 47 \%\right.$ ee $) ;{ }^{1} \mathrm{H} \mathrm{NMR}\left(\mathrm{CDCl}_{3}, 400\right.$ MHz) $\delta 1.02(\mathrm{t}, J=7.2,3 \mathrm{H}), 1.30(\mathrm{t}, J=7.2,3 \mathrm{H}), 3.30(\mathrm{dd}, J$ $=3.6,17.0,1 \mathrm{H}), 3.60(\mathrm{dd}, J=9.6,17.0,2 \mathrm{H}), 3.83(\mathrm{~s}, 3 \mathrm{H})$, $3.92-4.10(\mathrm{~m}, 2 \mathrm{H}), 4.30(\mathrm{q}, J=7.6,2 \mathrm{H}), 4.51(\mathrm{ddd}, J=3.2$, 9.8, 33.2 Hz, 1H), $6.87(\mathrm{~d}, J=8.8,2 \mathrm{H}), 7.17-7.24(\mathrm{~m}, 4 \mathrm{H})$, $7.35(\mathrm{~d}, J=8.4,1 \mathrm{H}), 7.85(\mathrm{~d}, J=9.2,2 \mathrm{H}) ;{ }^{13} \mathrm{C} \mathrm{NMR}$ $\left(\mathrm{CDCl}_{3}, 50 \mathrm{MHz}\right) \delta 194.4,167.9,167.1,137.5,131.7,129.5$, $128.5,128.4,128.2,128.0,127.8,127.5,127.0,126.0$, 125.8, 67.4, 67.2, 57.4, 42.2, 40.8; HRMS calcd for $\mathrm{C}_{23} \mathrm{H}_{25} \mathrm{FO}_{6} \mathrm{Na}\left([\mathrm{M}+\mathrm{Na}]^{+}\right)$439.1533, found 439.1533; $\mathrm{R}_{\mathrm{t}}$ HPLC (90 : 10, hexane : iso-PrOH, $254 \mathrm{~nm}, 1.0 \mathrm{~mL} / \mathrm{min}$ ) Whelk-O1 column, $t_{R}=24.5 \mathrm{~min}$ (major), $t_{R}=28.5 \mathrm{~min}$ (minor).

2-Fluoro-2-[3-(4-methoxyphenyl)-3-oxo-1-phenylpropyl] dibenzylmalonate (8d). $\mathrm{R}_{\mathrm{f}} 0.34($ EtOAc : hexane = $1: 4)$; $[\alpha]_{\mathrm{D}}^{25}-20.7\left(\mathrm{c}=1.0, \mathrm{CHCl}_{3}, 51 \%\right.$ ee $) ;{ }^{1} \mathrm{H} \mathrm{NMR}\left(\mathrm{CDCl}_{3}\right.$, 
$200 \mathrm{MHz}) \delta 3.16(\mathrm{dd}, J=3.5,17.5 \mathrm{~Hz}, 1 \mathrm{H}), 3.57(\mathrm{dd}, J=$ 9.8, $17.5 \mathrm{~Hz}, 1 \mathrm{H}), 3.85(\mathrm{~S}, 3 \mathrm{H}), 4.52$ (ddd, $J=3.5,9.8,33.1$ $\mathrm{Hz}, 1 \mathrm{H}), 4.90(\mathrm{~d}, J=3.2 \mathrm{~Hz}, 2 \mathrm{H}), 5.25(\mathrm{~d}, J=2.2 \mathrm{~Hz}, 2 \mathrm{H})$, $6.86(\mathrm{~d}, J=6.9 \mathrm{~Hz}, 2 \mathrm{H}), 7.03-7.08(\mathrm{~m}, 1 \mathrm{H}), 7.16-7.19(\mathrm{~m}$, $2 \mathrm{H}), 7.24-7.33(\mathrm{~m}, 12 \mathrm{H}), 7.79(\mathrm{~d}, J=6.8 \mathrm{~Hz}, 2 \mathrm{H})$; HRMS calcd for $\mathrm{C}_{33} \mathrm{H}_{29} \mathrm{FO}_{6} \mathrm{Na}\left([\mathrm{M}+\mathrm{Na}]^{+}\right) 563.1846$, found 563.1848; $\mathrm{R}_{\mathrm{t}} \operatorname{HPLC}(50: 50$, hexane : iso-PrOH, $254 \mathrm{~nm}, 1.0 \mathrm{~mL} / \mathrm{min})$ Chiralpak AS column, $t_{R}=8.6$ min (minor), $t_{R}=13.7 \mathrm{~min}$ (major).

2-[1-(4-Chlorophenyl)-3-oxo-3-phenylpropyl]-2-fluorodiethylmalonate (8e). $\mathrm{R}_{\mathrm{f}} 0.25$ (EtOAc : hexane $=1: 6$ ); $[\alpha]_{\mathrm{D}}^{25}-22.0\left(\mathrm{c}=1.0, \mathrm{CHCl}_{3}, 37 \%\right.$ ee $) ;{ }^{1} \mathrm{H} \mathrm{NMR}\left(\mathrm{CDCl}_{3}\right.$, $400 \mathrm{MHz}) \delta 1.07(\mathrm{t}, J=7.2,3 \mathrm{H}), 1.31(\mathrm{t}, J=7.2,3 \mathrm{H}), 3.36$ (dd, $J=3.2,17.6,1 \mathrm{H}), 3.62(\mathrm{dd}, J=10.0,17.6,1 \mathrm{H}), 3.96-$ 4.09 (m, 2H ), 4.31 (q, $J=7.2,2 \mathrm{H}), 4.50$ (ddd, $J=3.6,9.8$, $32.6 \mathrm{~Hz}, 1 \mathrm{H}), 7.21(\mathrm{~d}, J=8.4 \mathrm{~Hz}, 2 \mathrm{H}), 7.31(\mathrm{~d}, J=7.6 \mathrm{~Hz}$, $2 \mathrm{H}), 7.39-7.43(\mathrm{~m}, 2 \mathrm{H}), 7.52(\mathrm{~m}, 1 \mathrm{H}), 7.86(\mathrm{~d}, J=6.8 \mathrm{~Hz}$, $2 \mathrm{H}) ;{ }^{13} \mathrm{C}$ NMR $\left(\mathrm{CDCl}_{3}, 50 \mathrm{MHz}\right) \delta 196.7,168.7,167.7$, 130.2, 128.4, 127.1, 113.5, 67.2, 67.0, 57.5, 55.4, 41.9, 40.9; HRMS calcd for $\mathrm{C}_{22} \mathrm{H}_{22} \mathrm{FClO}_{5} \mathrm{Na}\left([\mathrm{M}+\mathrm{Na}]^{+}\right) 443.1037$, found 443.1039; $\mathrm{R}_{\mathrm{t}}$ HPLC (90: 10, hexane : iso-PrOH, 254 $\mathrm{nm}, 0.5 \mathrm{~mL} / \mathrm{min}$ ) Chiralcel OD-H column, $\mathrm{t}_{\mathrm{R}}=12.7 \mathrm{~min}$ (major), $\mathrm{t}_{\mathrm{R}}=14.6 \mathrm{~min}$ (minor).

2-[1-(4-Chlorophenyl)-3-oxo-3-phenylpropyl]-2-fluorodibenzylmalonate $(\mathbf{8 f}) . \mathrm{R}_{\mathrm{f}} 0.43($ EtOAc : hexane $=1: 4)$; $[\alpha]_{\mathrm{D}}^{25}-19.0\left(\mathrm{c}=1.0, \mathrm{CHCl}_{3}, 37 \%\right.$ ee $) ;{ }^{1} \mathrm{H} \mathrm{NMR}\left(\mathrm{CDCl}_{3}\right.$, $200 \mathrm{MHz}) \delta 3.16(\mathrm{dd}, J=3.2,17.8 \mathrm{~Hz}, 1 \mathrm{H}), 3.55$ (dd, $J=$ $10.2,17.8 \mathrm{~Hz}, 1 \mathrm{H}), 4.48$ (ddd, $J=3.2,10.2,32.8 \mathrm{~Hz}, 1 \mathrm{H})$, $4.96(\mathrm{~s}, 2 \mathrm{H}), 5.26(\mathrm{~d}, J=4.7 \mathrm{~Hz}, 2 \mathrm{H}), 7.03-7.10(\mathrm{~m}, 4 \mathrm{H})$, $7.17-7.32(\mathrm{~m}, 10 \mathrm{H}), 7.41(\mathrm{~d}, J=7.6 \mathrm{~Hz}, 2 \mathrm{H}), 7.52(\mathrm{~d}, J=7.2$ $\mathrm{Hz}, 1 \mathrm{H}), 7.77(\mathrm{~d}, J=7.0 \mathrm{~Hz}, 2 \mathrm{H})$; HRMS calcd for $\mathrm{C}_{32} \mathrm{H}_{26} \mathrm{FClO}_{5} \mathrm{Na}\left([\mathrm{M}+\mathrm{Na}]^{+}\right) 567.1350$, found 567.1347; $\mathrm{R}_{\mathrm{t}}$ HPLC (50:50, hexane : iso-PrOH, $254 \mathrm{~nm}, 0.7 \mathrm{~mL} / \mathrm{min}$ ) Chiralpak AS column, $t_{\mathrm{R}}=9.9 \mathrm{~min}(\operatorname{minor}), \mathrm{t}_{\mathrm{R}}=11.1 \mathrm{~min}$ (major).

2-[3-(3-Bromophenyl)-1-(4-chlorophenyl)-3-oxo-propyl]2-fluorodiethylmalonate (8g). $\mathrm{R}_{\mathrm{f}} 0.22($ EtOAc : hexane $=$ $1: 6) ;[\alpha]_{\mathrm{D}}^{25}-18.7\left(\mathrm{c}=1.0, \mathrm{CHCl}_{3}, 37 \%\right.$ ee $) ;{ }^{1} \mathrm{H}$ NMR $\left(\mathrm{CDCl}_{3}, 400 \mathrm{MHz}\right) \delta 1.07(\mathrm{t}, J=7.2 \mathrm{~Hz}, 3 \mathrm{H}), 1.32(\mathrm{t}, J=7.2$ $\mathrm{Hz}, 3 \mathrm{H}), 3.35$ (dd, $J=3.2,17.6 \mathrm{~Hz}, 1 \mathrm{H}), 3.56$ (dd, $J=10.0$, $17.6 \mathrm{~Hz}, 1 \mathrm{H}), 3.97-4.10(\mathrm{~m}, 2 \mathrm{H}), 4.31(\mathrm{q}, J=7.6 \mathrm{~Hz}, 2 \mathrm{H})$, 4.47 (ddd, $J=3.6,9.8,32.4 \mathrm{~Hz}, 1 \mathrm{H}), 7.20-7.31(\mathrm{~m}, 5 \mathrm{H})$, 7.65 (d, $J=8.0 \mathrm{~Hz}, 1 \mathrm{H}), 7.79$ (d, $J=8.0 \mathrm{~Hz}, 1 \mathrm{H}), 7.97$ (s, $1 \mathrm{H}) ;{ }^{13} \mathrm{C} \mathrm{NMR}\left(\mathrm{CDCl}_{3}, 50 \mathrm{MHz}\right) \delta 195.3,167.9,167.3$, $139.8,134.9,133.6,132.0,128.5,128.4,128.2,128.1$, 127.2, 67.3, 67.1, 57.3, 42.9, 41.0; HRMS calcd for $\mathrm{C}_{22} \mathrm{H}_{21} \mathrm{BrClFO}_{5} \mathrm{Na}\left([\mathrm{M}+\mathrm{Na}]^{+}\right)$521.0143, found 521.0139; $\mathrm{R}_{\mathrm{t}}$ HPLC (90 : 10, hexane : iso-PrOH, $254 \mathrm{~nm}, 0.5 \mathrm{~mL} / \mathrm{min}$ ) Chiralcel OD-H column, $\mathrm{t}_{\mathrm{R}}=12.8$ min (major), $\mathrm{t}_{\mathrm{R}}=15.6$ $\min ($ minor).

2-[3-(3-Bromophenyl)-1-naphthalen-2-yl-3-oxopropyl]2-fluorodiethylmalonate (8h). $\mathrm{R}_{\mathrm{f}} 0.19$ (EtOAc : hexane $=$ $1: 6) ;[\alpha]_{\mathrm{D}}^{25}-19.7\left(\mathrm{c}=1.0, \mathrm{CHCl}_{3}, 47 \%\right.$ ee); ${ }^{1} \mathrm{H}$ NMR $\left(\mathrm{CDCl}_{3}, 400 \mathrm{MHz}\right) \delta 0.94(\mathrm{t}, J=7.2,3 \mathrm{H}), 1.33(\mathrm{t}, J=7.2$, $3 \mathrm{H}), 3.45(\mathrm{dd}, J=3.2,17.6 \mathrm{~Hz}, 1 \mathrm{H}), 3.72(\mathrm{dd}, J=9.6,17.4$ $\mathrm{Hz}, 1 \mathrm{H}), 3.88-3.98(\mathrm{~m}, 2 \mathrm{H}), 4.33(\mathrm{q}, J=7.6 \mathrm{~Hz}, 2 \mathrm{H}), 4.67$ (ddd, $J=3.6,9.6,32.8 \mathrm{~Hz}, 1 \mathrm{H}), 7.26(\mathrm{~d}, J=15.6 \mathrm{~Hz}, 1 \mathrm{H})$,
7.40-7.42 (m, 2H), $7.50(\mathrm{~d}, J=8.4 \mathrm{~Hz}, 1 \mathrm{H}), 7.61(\mathrm{~d}, J=8.0$ $\mathrm{Hz}, 1 \mathrm{H}), 7.72-7.81(\mathrm{~m}, 5 \mathrm{H}), 7.97(\mathrm{~s}, 1 \mathrm{H}) ;{ }^{13} \mathrm{C} \mathrm{NMR}\left(\mathrm{CDCl}_{3}\right.$, $50 \mathrm{MHz}) \delta 197.5,168.0,167.5,136.6,135.1,132.9,132.0$, $129.1,128.4,128.3,128.1,128.0,113.7,67.2,67.0,57.7$, 55.0, 42.4, 40.1; HRMS calcd for $\mathrm{C}_{26} \mathrm{H}_{24} \mathrm{BrFO}_{5} \mathrm{Na}\left([\mathrm{M}+\mathrm{Na}]^{+}\right)$ 537.0689 , found $537.0681 ; \mathrm{R}_{\mathrm{t}}$ HPLC $(90: 10$, hexane : iso$\mathrm{PrOH}, 254 \mathrm{~nm}, 0.5 \mathrm{~mL} / \mathrm{min})$ Chiralcel OD-H column, $\mathrm{t}_{\mathrm{R}}=$ $14.8 \mathrm{~min}$ (major), $\mathrm{t}_{\mathrm{R}}=18.8 \mathrm{~min}$ (minor).

Acknowledgement. This work was supported by research grant from Soonchunhyang University.

\section{References}

1. (a) Dehmlow, E. V.; Dehmlow, S. S. Phase Transfer Catalysis, 3rd ed.; VCH: Weinheim, 1993. (b) Goldberg, Y. Phase Transfer Catalysis: Selected Problems and Application; Gordon \& Breach Science, Reading: 1992. (c) Starks, C. M.; Liotta, C. L.; Halpern, M. Phase Transfer Catalysis: Fundamentals, Applications, and Industrial Perspectives; Chapman \& Hall, New York, 1994.

2. For recent reviews, see: (a) Shioiri, T. In Handbook of PhaseTransfer Catalysis; Sasson, Y., Neumann. R., Eds.; Blackie Academic \& Professional: London, 1997; Ch. 14. (b) Shioiri, T.; Arai, S. In Stimulating Concepts in Chemistry; Vogtle. F., Stoddart, J. F., Shibasaki, M., Eds.; Wiley-VCH: Weinheim, 2000; p 123. (c) O'Donnell, M. J. In Catalytic Asymmetric Synthesis, 2nd ed.; Ojima, I., Ed.; Wiley-VCH: New York, 2000; Ch. 10. (d) O'Donnell, M. J. Aldrichchimica Acta 2001, 34, 3. (e) Maruoka, K.; Ooi, T. Chem. Rev. 2003, 103, 3013. (f) O'Donnell, M. J. Acc. Chem. Res. 2004, 37, 506. (g) Lygo, B.; Andrews, B. I. Acc. Chem. Res. 2004, 37, 518.

3. (1) For recent reviews and monographs see: (a) Leonard, J. Contemp. Org. Synth. 1994, 1, 387. (b) Perlmutter, P. Conjugate Addition Reactions in Organic Synthesis; Pergamon: Oxford, 1992. (c) Rossiter, B. E.; Swingle, N. M. Chem. Rev. 1992, 92, 771.

4. (a) Dieter, R. K.; Alexander, C. W.; Nice, L. E. Tetrahedron 2000 56, 2767. (b) Park, Y. S.; Weisenburger, G. A.; Beak, P. J. Am. Chem. Soc. 1997, 199, 10537. (c) Beak, P.; Lee, W. K. J. Org. Chem. 1993, 58, 1109. (d) Shawe, T. T.; Meyers, A. I. J. Org. Chem. 1991, 56, 2751. (e) Patrocinio, V. L.; Costa, P. R. R.; Correia, C. R. D. Synthesis 1994, 474. (f) Sawamura, H.; Hamashima, H.; Ito, Y. J. Am. Chem. Soc. 1992, 114, 8295.

5. (a) Arai, T.; Sasai, H.; Aoe, K.; Okamura, K.; Date, T.; Shibasaki, M. Angew. Chem. Int. Ed. Engl. 1996, 35, 104. (b) Funabashi, K.; Saida, Y.; Kanai, M.; Arai, T.; Sasai, H.; Shibasaki, M. Tetrahedron Lett. 1998, 39, 7557. (c) Shimizu, S.; Ohori, K.; Arao, T.; Sasai, H.; Shibasaki, M. J. Org. Chem. 1998, 63, 7547. (d) Kim, Y. S.; Matsunaga, S.; Das, J.; Sekine, A.; Ohshima, T.; Shibasaki, M. J. Am. Chem. Soc. 2000, 122, 6506. (e) Takita, R.; Ohshima, T.; Shibasaki, M. Tetrahedron Lett. 2002, 43, 4661. (f) Anamalai, V.; DiMauro, E. F.; Carroll, P. J.; Kozlowski, M. C. J. Org. Chem. 2003, 63, 1973. (g) Kumaraswamy, G.; Jena, N.; Sastrys, M. N. V.; Venkata Ras, G.; Ankamma, K. J. Mol. Cat. A 2005, 230, 39 .

6. (a) Yamaguchi, M.; Igarashi, Y.; Reddy, R. S.; Shiraishi, T.; Hirama, M. Tetrahedron 1997, 53, 11223. (b) Yamaguchi, M.; Shiraishi, T.; Igarashi, Y.; Hirama, M. Tetrahedron Lett. 1994, 25, 823. (c) Yamaguchi, M.; Shiraishi, T.; Hirama, M. J. Org. Chem. 1996, 61, 3520. (d) Hanessian, S.; Pham, V. Org. Lett. 2000, 2 , 2975.

7. (a) Narasimhan, S.; Balakumar, V. R.; Radhakrishnan, V. Tetrahedron Lett. 2001, 42, 719. (b) Sundarajan, G.; Prabagaran, N. Org. Lett. 2001, 3, 389. (c) DiMauro, E. F.; Kozlowski, M. C. Org. Lett. 2001, 3, 1641. (d) Jha, S. C.; Joshi, N. N. Tetrahedron: Asymmetry 2001, 12, 2463. (e) MacCulloch, A. C.; Yolka, S.; 
Jackson, R. F. W. Synlett 2002, 1700.

8. Kawara, A.; Taguchi, T. Tetrahedron Lett. 1994, 35, 8805.

9. (a) Perrard, T.; Plaquevent, L.-C.; Desmurs, J.-R.; Hebrault, D. Org. Lett. 2000, 2, 2959. (b) Ooi, T.; Ohara, D.; Fukumoto, K.; Maruoka, K. Org. Lett. 2005, 7, 3195.

10. (a) Kim, D. Y.; Huh, S. C. Tetrahedron 2001, 57, 8933. (b) Kim, D. Y.; Park, E. J. Org. Lett. 2002, 4, 545. (c) Kim, D. Y.; Choi, Y. J.; Park, H. Y.; Joung, C. U.; Koh, K. O.; Mang, J. Y.; Jung, K.-Y. Synth. Commun. 2003, 33, 435. (d) Park, E. J.; Kim, H. R.; Joung, C. W.; Kim, D. Y. Bull. Korean Chem. Soc. 2004, 25, 1451. (e) Kim, D. Y.; Huh, S. C. Bull. Korean Chem. Soc. 2004, 25, 347. (f) Park, E. J.; Kim, M. H.; Kim, D. Y. J. Org. Chem. 2004, 69, 6897. (g) Kim, H. R.; Kim, D. Y. Tetrahedron Lett. 2005, 46, 3115. (h) Kim, S. M.; Kim, H. R.; Kim, D. Y. Org. Lett. 2005, 7, 2309. (i) Kim, S. M.; Kang, Y. K.; Lee, K.; Mang, J. Y.; Kim, D. Y. Bull.
Korean Chem. Soc. 2006, 26, 423. (j) Kang, Y. K.; Kim, D. Y. Tetrahedron Lett. 2006, 47, 4565.

11. (a) Kim, D. Y.; Huh, S. C.; Kim, S. M. Tetrahedron Lett. 2001, 42, 6299. (b) Kim, D. Y.; Kim, S. M.; Koh, K. O.; Mang, J. Y. Bull. Korean Chem. Soc. 2003, 24, 1425.

12. Park, B. S.; Kim, D. Y.; Rosenthal, P. J.; Huh, S. C.; Lee, B. J.; Park, S. M.; Kim, J. E.; Kim, M. H.; Huh, T. L.; Choi, Y, J.; Suh, K. H.; Choi, W. S.; Lee, S. E. Bioorg. Med. Chem. Lett. 2002, 12, 1351.

13. (a) Hiyama, T.; Kanie, K.; Kusumoto, T.; Morizawa, Y.; Shimizu, M. Organofluorine Compounds: Chemistry and Applications; Springer-Verlag: Berlin, 2000. (b) Biomedical Frontiers of Fluorine Chemistry; Ojima, I., McCarthy, J. R., Welch, J. T., Eds.; ACS Symposium Series 639; American Chemical Society: Washington, DC, 1996. 